Check for updates

Cite this: RSC Adv., 2019, 9, 7324

Received 17th January 2019

Accepted 15th February 2019

DOI: $10.1039 / c 9 r a 00433 e$

rsc.li/rsc-advances

\section{Facile fabrication of long-chain alkyl functionalized ultrafine reduced graphene oxide nanocomposites for enhanced tribological performance $\uparrow$}

\author{
Chao Zhu, Yehai Yan, (D) Fan Wang, Jian Cui, Shuai Zhao, (D) Ailin Gao \\ and Guangfa Zhang (iD) *
}

Due to their ultrathin 2D laminated structure as well as excellent mechanical and thermal stabilities, ultrafine graphene-based nanoparticles exhibit fascinating advantages as highly-efficient lubricant additives. However, it remains a daunting challenge to achieve good and durable dispersion of these graphene-based nanoparticles in lubricating oils. Herein, we report a facile and efficient integration strategy involving particle size miniaturization, surface grafting with octadecyl alcohol (OA), and partial chemical reduction to prepare a novel long-chain alkyl functionalized ultrafine reduced graphene oxide (RGO-g-OA) with highlydispersive capacity and superior tribological performance. The chemical composition and structural characteristics, microstructural morphology, and particle size distribution of RGO-g-OA were systematically investigated. Combining significantly improved lipophilicity derived from the long-chain alkyl grafting and partial chemical reduction with the small-size effect gave rise to outstanding long-term dispersion stability (as long as one month) of RGO-g-OA in the finished oil. Moreover, the friction coefficient and wear volume of finished oil with merely $0.005 \mathrm{wt} \% \mathrm{RGO}-\mathrm{g}$-OA greatly reduced to 0.065 and $10316 \mu \mathrm{m}^{3}$, decreased by $9.7 \%$ and $44 \%$, respectively, compared to those of pristine finished oil, demonstrating remarkable friction reduction and anti-wear performances. Consequently, owing to the characteristics of facile fabrication, durable dispersion stability, and superior tribological performance at an extremely low content, this novel nanoadditive shows a promising application potential in the tribology field.

\section{Introduction}

The friction and wear phenomena are ubiquitous between various adjacent mechanical surfaces, such as automobile engines and bearings, and generally lead to undesirable energy dissipation, decreased lifetime of mechanical components, and even irrevocable catastrophic failure. ${ }^{1-4}$ Utilization of lubricant oil is considered one of the most effective methods to reduce or even eliminate the destructive friction and wear issues. However, with work parameters such as loading, speed, and temperature further ascending, conventional lube oils are incapable of fulfilling the rigorous requirements of frictionreduction and wear-resistance for modern mechanical equipment. ${ }^{5,6}$ Therefore, novel high performance lubricants with stronger friction and wear reduction capacity are required.

Encouragingly, in recent years, employment of nanomaterials as superior solid lubricants or nanoadditives has

Key Laboratory of Rubber-Plastics, Ministry of Education/Shandong Provincial Key Laboratory of Rubber-Plastics, School of Polymer Science and Engineering, Qingdao University of Science and Technology, Qingdao 266042, P. R. China. E-mail: gfzhang@qust.edu.cn

$\dagger$ Electronic supplementary information (ESI) available. See DOI: 10.1039/c9ra00433e attracted extensive attention due to their unique merits, such as smaller size and chemical and thermal stability., ${ }^{4,7}$ These nanomaterials can not only easily form a shearing thin film at the sliding contact interface and thus yield low friction, but also fill and/or repair the grooves formed on the worn surfaces, revealing comprehensive advantages for improving the tribological performances of lube oil. Graphene, as an emerging star of nano-lubricating oil additives, exhibits irresistible advantages in the area of tribology, owning to its inherently unique structural characteristics. ${ }^{8}$ Specifically, smaller size and ultrathin lamellar structure make it easily enter the mated interfaces of friction pairs, avoiding the direct contact of rubbing surfaces and thus greatly reducing friction and wear during friction processes. ${ }^{8}$ Moreover, excellent mechanical and thermal stability ensure that graphene can maintain a more stable and highly-efficient lubricating performance even under harsh tribology conditions, i.e., high pressure, high temperature, and high mechanical stresses. ${ }^{4}$ Based on these advantages, considerable efforts have been devoted to exploit graphene as a nanoadditive for enhanced friction reduction and anti-wear properties. $^{8-10}$ For example, Eswaraiah et al. dispersed graphene in engine oil by probe sonication and an elevated lubricating performance was observed: the friction coefficient and wear scar diameter were reduced by $80 \%$ and $33 \%$, 
respectively. ${ }^{11}$ Zhao et al. reported a polyalphaolefin lubricant oil with added graphene, which showed 30\% decrease of the friction coefficient and few scratches on the rubbing surface. ${ }^{12}$ Although pristine graphene as an additive can improve the tribology properties of lubricating oil to some extent, the easy aggregation behavior of pristine graphene is a troublesome obstacle, severely restricting its widespread application. ${ }^{13}$ To overcome this limitation, the introduction of additional molecular dispersants and surface chemical modification are the two most common strategies for enhancing the dispersion capability of pristine graphene. ${ }^{\mathbf{4}}$ However, these methods suffer from some distinct disadvantages including the following: (a) unstable molecular additives tend to decompose because of the high shear stresses and high local temperatures at the rubbing surfaces during the friction process; (b) due to the strong chemical inertness of pristine graphene, it is quite difficult to directly conduct covalent functional modification (such as surface grafting) onto its surface..$^{15}$

Fortunately, graphene oxide (GO), a vital graphene derivative, has become a promising candidate as an effective lubricant additive because of its intriguing significant advantages: ${ }^{\mathbf{1 6 , 1 7}}$ (I) it features $2 \mathrm{D}$ single atom layered structure and excellent mechanical properties; (II) its mature manufacturing technology leads to a possibility of large-scale production and low cost for practical application; (III) abundant oxygen-containing groups (such as carboxyl, hydroxyl, and epoxy groups) on GO's surface provide the crucial chemical basis for further chemical grafting modification. ${ }^{18}$ However, it should be pointed out that pure GO is not suitable for direct utilization in lubricating oils since abundant oxygenated components can greatly decrease its inherent thermal stability and also distinctly weaken its dispersion capacity in non-polar lubricant oils. ${ }^{19}$ In recent years, many methodologies have been explored to address these issues. ${ }^{20-24}$ Among them, surface grafting of long-chain non-polar groups onto GO is regarded as one of the most efficient strategies to reinforce its lipophilicity and thus increase its dispersion stability in lube oils. ${ }^{25-29}$ For instance, Shivina et al. prepared an octadecylamine grafted graphene oxide, which exhibits good dispersion capacity in hexadecane, by coupling alkylamine with the carboxylic groups in GO. ${ }^{30}$ Nurul et al. demonstrated that highly oil-dispersed GO sheets can be fabricated by introducing an organic moiety using CuAAC via click chemistry; the base oil formulated with these functionalized GO additives achieves good friction-reducing and anti-wear properties. ${ }^{28}$ Note that the particle size of graphene-based nanomaterials has great influence on their dispersion stability. ${ }^{31}$ However, dispersibility and tribological performance both affected by the synergistic effect between chemical modification and particle size of GO have been rarely reported so far.

Herein, we demonstrate an efficient integrated strategy for fabricating novel small-size and alkyl-functionalized reduced graphene oxide (RGO-g-OA) with high dispersion capacity and superior tribological performance. As illustrated in Scheme 1, the preparation procedure included three key steps: (a) mechanical crushing to yield smaller-sized GO; (b) esterification reaction using octadecyl alcohol (OA); (c) partial chemical reduction by L-ascorbic acid. The chemical composition, structural characteristics, microscopic morphology, and particle size of RGO-g-OA were comprehensively investigated. Interestingly, the resultant RGO- $g$-OA exhibited excellent thermal stability and long-term dispersion stability (as long as one month) in the finished oil. Moreover, the tribological performance of finished oil formulated with different weight fractions of RGO- $g$-OA was systematically evaluated by a reciprocating friction and wear method. A possible corresponding frictionreducing and anti-wear mechanism was discussed as well.

\section{Experimental}

\subsection{Materials}

Natural graphite (purity > 99\%, 300 mesh) was purchased from Qingdao Haida Co., Ltd. Concentrated sulfuric acid $\left(\mathrm{H}_{2} \mathrm{SO}_{4}\right.$,

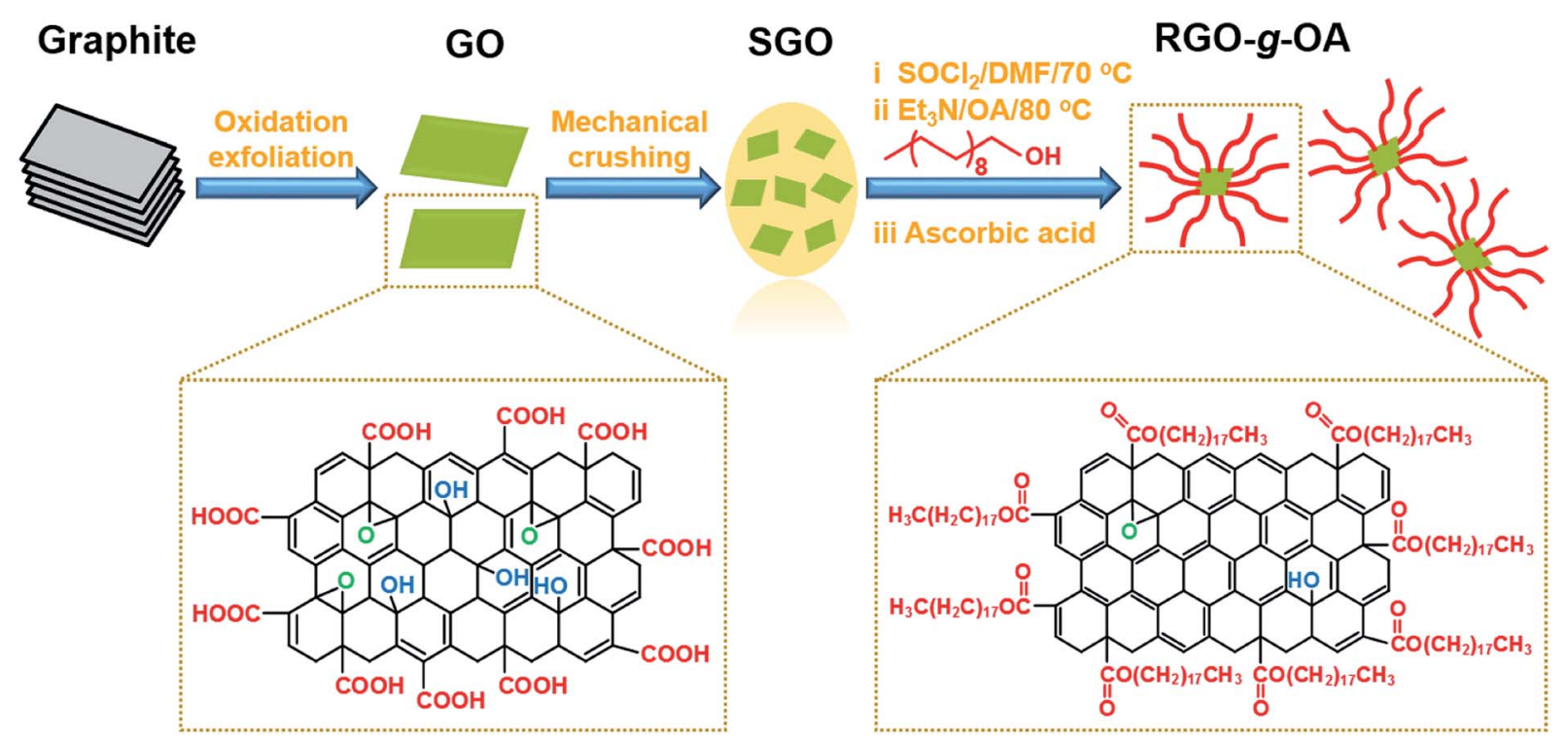

Scheme 1 Schematic of preparation procedures for RGO-g-OA, involving the $\mathrm{SOCl}_{2}$ pre-treatment of GO (step I), the esterification reaction between $\mathrm{OA}$ and acyl-chloride functionalized GO (step II), and partial chemical reduction utilizing L-ascorbic acid (step III). 
98\%), $N, N$-dimethyl formamide (DMF), L-ascorbic acid, sodium nitrate $\left(\mathrm{NaNO}_{3}\right)$, potassium permanganate $\left(\mathrm{KMnO}_{4}\right)$, hydrogen peroxide $\left(\mathrm{H}_{2} \mathrm{O}_{2}, 30 \%\right)$, hydrochloric acid ( $\left.\mathrm{HCl}, 37 \%\right)$, deuterated dimethyl sulfoxide (DMSO), and tetrahydrofuran (THF) were purchased from Sinopharm Chemical Reagent Co., Ltd. Ultrapure water (18.25 $\mathrm{M} \Omega$ ) was produced in the laboratory using ULTRAPURE (UPHeI-20T). Octadecyl alcohol (OA) was obtained from Tianjin Bodi Co., Ltd. Thionyl chloride $\left(\mathrm{SOCl}_{2}\right)$ and triethylamine $\left(\mathrm{Et}_{3} \mathrm{~N}\right)$ were supplied by Tianjin Fuyu Fine Chemical Co., Ltd. The finished oil used in this work was synthesized in the laboratory and detailed recipe information is listed in Table $\mathrm{S} 1 . \dagger$ The hydraulic oil (kinematic viscosity $13.2 \mathrm{~mm}^{2} \mathrm{~s}^{-1}$ at 40 ${ }^{\circ} \mathrm{C}$ ) utilized as the base oil was provided by Kunlun Lubricant, China.

\subsection{Preparation of chemically grafting reduced graphene oxide (RGO-g-OA)}

As illustrated in Scheme 1, graphene oxide (GO) was prepared based on a modified Hummers' method, which is specifically described in the ESI. $\dagger$ The resulting GO was further fragmented by ultrasonic treatment for $15 \mathrm{~min}$ and high-speed dispersion with a homogenizer (XHF-DY) at $4500 \mathrm{rpm}$ for $15 \mathrm{~min}$ (repeated three times), to yield smaller-sized graphene oxide (SGO). Then, chemically covalent functionalized reduced graphene oxide was fabricated by a facile two-step method: (1) surface grafting via an esterification reaction using octadecyl alcohol; (2) partial chemical reduction by L-ascorbic acid.

Typically, GO (50 mg) and DMF (2 mL) were added to a round-bottom flask $(50 \mathrm{~mL})$ and mixed by ultrasonication for $30 \mathrm{~min}$, followed by $\mathrm{SOCl}_{2}(20 \mathrm{~mL})$ being slowly added to the mixture. The acyl chloride reaction was then conducted at $70{ }^{\circ} \mathrm{C}$ for $24 \mathrm{~h}$ and the resultant product was washed with DMF by centrifugation three times to obtain acyl chlorinated graphene oxide (GO-COCl). Afterwards, triethylamine $\left(\mathrm{Et}_{3} \mathrm{~N}, 2\right.$ $\mathrm{mL}$ ) and $\mathrm{OA}(3 \mathrm{~g})$ were added into the GO-COCl/DMF dispersion $\left(2.5 \mathrm{mg} \mathrm{mL}^{-1}, 20 \mathrm{~mL}\right)$ in an ice-water bath and the grafting reaction was conducted under reflux at $80{ }^{\circ} \mathrm{C}$ for $24 \mathrm{~h}$. The obtained mixture was filtered to yield long-chain alkylated graphene oxide (GO-g-OA). Finally, a certain amount of L-ascorbic acid (weight ratio $\mathrm{L}$-ascorbic acid/GO- $g$-OA $=2: 1$ ) was added to partially chemically reduce GO- $g$-OA at $80^{\circ} \mathrm{C}$ for $6 \mathrm{~h}$, followed by filtration washing with DMF and drying at $60{ }^{\circ} \mathrm{C}$ in an oven to acquire chemically reduced alkylated graphene oxide (RGO- $g$ OA). For comparison, reduced graphene oxide (RGO) treated by L-ascorbic acid without alkylate functionalization was also prepared.

\subsection{Preparation of lubricant oil samples}

A certain amount of the obtained RGO- $g$-OA was first completely dissolved in THF $\left(2.5 \mathrm{mg} \mathrm{mL}^{-1}\right)$ by ultrasonication for $30 \mathrm{~min}$. The additive mixture was then mixed with lubricating oil (finished oil or base oil) using magnetic stirring (500 rpm) for $30 \mathrm{~min}$. After sufficient mixing, the THF was thoroughly removed with a rotary evaporator at $50{ }^{\circ} \mathrm{C}$. The weight concentrations of RGO- $g$-OA in the lubricating oils were $0 \mathrm{wt} \%$, $0.005 \mathrm{wt} \%, 0.010 \mathrm{wt} \%$, and $0.025 \mathrm{wt} \%$.

\subsection{Tribological property test}

Tribological tests were carried out with an Optimol SRV oscillating reciprocating friction and wear tester. The experimental apparatus is visually depicted in Scheme 2 . The diameter of the GCr15 bearing steel ball is $10 \mathrm{~mm}$ and the surface roughness is $\sim 20 \mathrm{~nm}$. The surface roughness of GCr15 bearing steel disc $(24 \mathrm{~mm} \times 8 \mathrm{~mm})$ is about $40 \mathrm{~nm}$. To remove organic contaminants, all the friction pairs were cleaned in petroleum ether by ultrasonication before tests. About $25 \mu \mathrm{L}$ homemade finished oil or base oil with different concentrations of additive was added to the disc surface. A prescribed $30 \mathrm{~s}$ running-in period before formal testing was performed to prevent friction pair system damage. The load was initially set at $50 \mathrm{~N}$ and then slowly increased to the final value. During the test, all samples used the same conditions: stroke of $1 \mathrm{~mm}$, frequency $25 \mathrm{~Hz}$, load of 100 or $150 \mathrm{~N}$, and temperature at $50{ }^{\circ} \mathrm{C}$. The friction coefficient values were recorded automatically by a recorder attached to the SRV tester. After the tests, the friction pairs were cleaned with petroleum ether by ultrasonication $(10 \mathrm{~min})$ to remove residual lubricant oils and, occasionally, accumulated debris. Then, the volumes of wear scars on the discs were measured by a MicroXAM 3D noncontact surface mapping profiler (equipped with Apex analysis software).

\subsection{Characterization}

Fourier transform infrared spectra (FTIR) were recorded using a Bruker VERTEX 70 spectrometer in wavenumber range of 4000-400 $\mathrm{cm}^{-1}$. ${ }^{1} \mathrm{H}$ NMR spectra $(400 \mathrm{MHz})$ were measured on a Bruker Advance spectrometer using deuterated DMSO as the solvent. The Raman spectra were obtained using a Renishaw Invia Raman Microscope with $532 \mathrm{~nm}$ laser excitation wavelength at room temperature. The X-ray diffraction (XRD) patterns were examined using a Bruker D8 X-ray diffractometer with a $2 \theta$ angle ranging from 5 to $60^{\circ}$ and a scan rate of $10^{\circ} \mathrm{min}^{-1}$. Transmission electron microscopy (TEM) was probed using a JEOL JEM-2100 microscope with $200 \mathrm{kV}$ accelerating

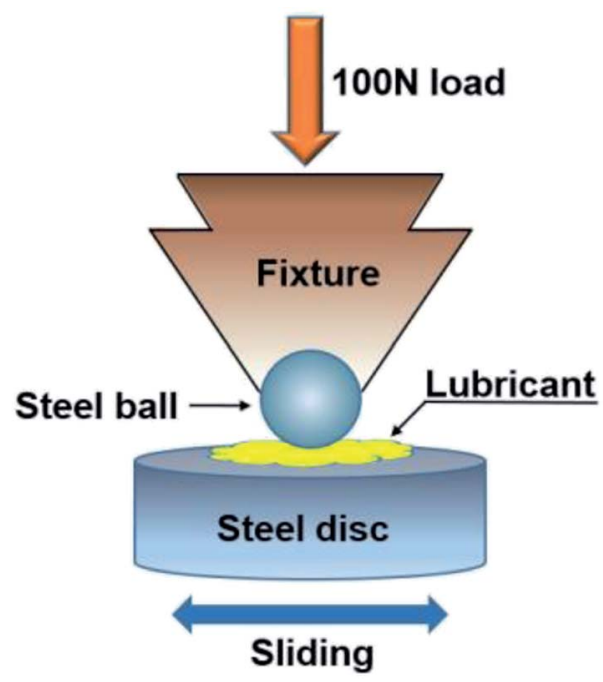

Scheme 2 Schematic of the tribological performance measurement apparatus. 
voltage. Particle size distributions of the original GO and RGO- $g$ OA nanosheets were observed dispersed in a certain amount of ethanol medium by dynamic light scattering (DLS) with a Malvern Zetasizer Nano ZS90. Thermogravimetric analysis (TGA) was carried out with a TA Instruments Q50 thermogravimetric analyzer under nitrogen atmosphere in the range of $50-800{ }^{\circ} \mathrm{C}$ at a heating range of $10{ }^{\circ} \mathrm{C} \mathrm{min}^{-1}$.

\section{Results and discussion}

\subsection{Chemical composition characterization}

To identify the chemical composition evolution of GO, GO- $g$-OA, and RGO-g-OA, FTIR characterization of these materials was performed. As indicated in Fig. 1a, all typical characteristic peaks of GO can be clearly observed: C-O-C $\left(\sim 1050 \mathrm{~cm}^{-1}\right)$, carboxyl $\mathrm{C}-\mathrm{OH}\left(1404 \mathrm{~cm}^{-1}\right), \mathrm{C}=\mathrm{C}\left(1650 \mathrm{~cm}^{-1}\right)$ and $\mathrm{C}=\mathrm{O}$ $\left(1720 \mathrm{~cm}^{-1}\right)$ bonds. The $\mathrm{O}-\mathrm{H}$ stretching vibrations over the region of $3700-3300 \mathrm{~cm}^{-1}$ should be attributed to the hydroxyl and carboxyl groups of GO. This result reveals that a large number of oxygen-containing functional groups, such as carboxyl, hydroxyl, and epoxy groups, were successfully incorporated into the basal plane and edge of GO. It is known that carboxyl groups are mainly distributed on the edge of GO, while other oxygenated functional groups, like hydroxyl and epoxy groups, are generally immobilized onto the plane surface of GO. ${ }^{32}$ In comparison, obvious characteristic peaks at 2927 and $2850 \mathrm{~cm}^{-1}$ are found for GO- $g$-OA, ascribed to the typical stretching vibrations of long-chain alkyl groups $\left(-\mathrm{CH}_{2}\right.$ and $\left.-\mathrm{CH}_{3}\right)$. In addition, the peak strength of carboxyl groups $(\mathrm{C}=\mathrm{O}$, $1720 \mathrm{~cm}^{-1}$ ) in GO-g-OA exhibits a conspicuous decline compared to that of GO. Thus, we conclude that OA was successfully grafted onto the surface of GO. Moreover, for RGO$g$-OA, it is observed that the peak strength of $\mathrm{C}=\mathrm{O}$ shows a further discernable decrease compared with that of GO- $g$-OA. Simultaneously, the broad epoxide (C-O-C) peak becomes a narrow spike. These results may originate from the effective removal of the great mass of oxygen functional groups due to the chemical reduction process with L-ascorbic acid.

Additionally, ${ }^{1} \mathrm{H}$ NMR measurement was performed to validate the chemical structure of RGO-g-OA. As shown in Fig. 1b, four different chemical shifts, that is, at $0.85 \mathrm{ppm}\left(\mathrm{a},-\mathrm{CH}_{3}\right)$,
$1.23 \mathrm{ppm}\left(\mathrm{b},-\mathrm{CH}_{2}\right), 1.76 \mathrm{ppm}\left(\mathrm{c},-\mathrm{OCH}_{2} \mathrm{CH}_{2}-\right)$, and $4.32 \mathrm{ppm}(\mathrm{d}$, $-\mathrm{OCH}_{2} \mathrm{CH}_{2}-$ ) can be observed, further demonstrating that the long-alkyl chain OA were successfully grafted onto GO. Furthermore, strong chemical shift at $3.35 \mathrm{ppm}$ should be ascribed to other hydrogen protons located on the basal plane of RGO. Therefore, the achievements of long-chain alkane grafting and subsequent partial chemical reduction pave the way toward improved lipophilicity and dispersion stability of RGO- $g$-OA additive in lubricants.

\subsection{Structure characterization}

Generally, some structural defects are inevitably introduced onto the surfaces of carbon-based materials such as GO and RGO during their preparation processes. Raman spectroscopy is commonly regarded as a powerful and effective tool to evaluate the structural integrity of these carbonaceous materials. ${ }^{33}$ Fig. 2a presents the Raman spectra of GO, GO- $g$-OA, and RGO- $g$-OA. All samples display two distinct peaks: the D-band at $\sim 1350 \mathrm{~cm}^{-1}$ and the G-band at $\sim 1590 \mathrm{~cm}^{-1}$. The G-band represents $\mathrm{sp}^{2}$ carbon structures (graphitic carbon), originating from the inplane vibration of carbon atoms. Meanwhile, the D-band is associated with $\mathrm{sp}^{3}$ carbon structure originating from structural defects or partially disordered graphitic domains. Additionally, the intensity ratio of D-band to G-band $\left(I_{\mathrm{D}} / I_{\mathrm{G}}\right)$ has been extensively utilized as a typical parameter for evaluating the defect degree of graphene and its derivatives. ${ }^{34}$ The Raman spectra results show that the $I_{\mathrm{D}} / I_{\mathrm{G}}$ ratios increase from 1.32 for GO to 1.65 for GO- $g$-OA, indicating that extra defects were introduced onto the GO surface due to the esterification reaction. Interestingly, after subsequent treatment with L-ascorbic acid, the $I_{\mathrm{D}} / I_{\mathrm{G}}$ ratio decreases from 1.65 for GO- $g$-OA to 1.56 for RGO- $g$-OA, suggesting that some defects on the GO-g-OA surface were partially repaired because of the chemical reduction process.

The phase structures of GO, GO- $g$-OA, and RGO- $g$-OA were also examined by XRD analysis. As can be seen from Fig. $2 b$, for the XRD pattern of GO, an obvious (001) diffraction peak at $2 \theta=$ $11.8^{\circ}$ is found, with an interlayer space ( $d$-spacing) of $0.730 \mathrm{~nm}$ calculated by the Bragg equation. ${ }^{35}$ Compared with GO, GO- $g$ OA shows a new broad diffraction peak (002) at $2 \theta=23.6^{\circ}$, corresponding to $d$-spacing of $0.373 \mathrm{~nm}$, which is derived from
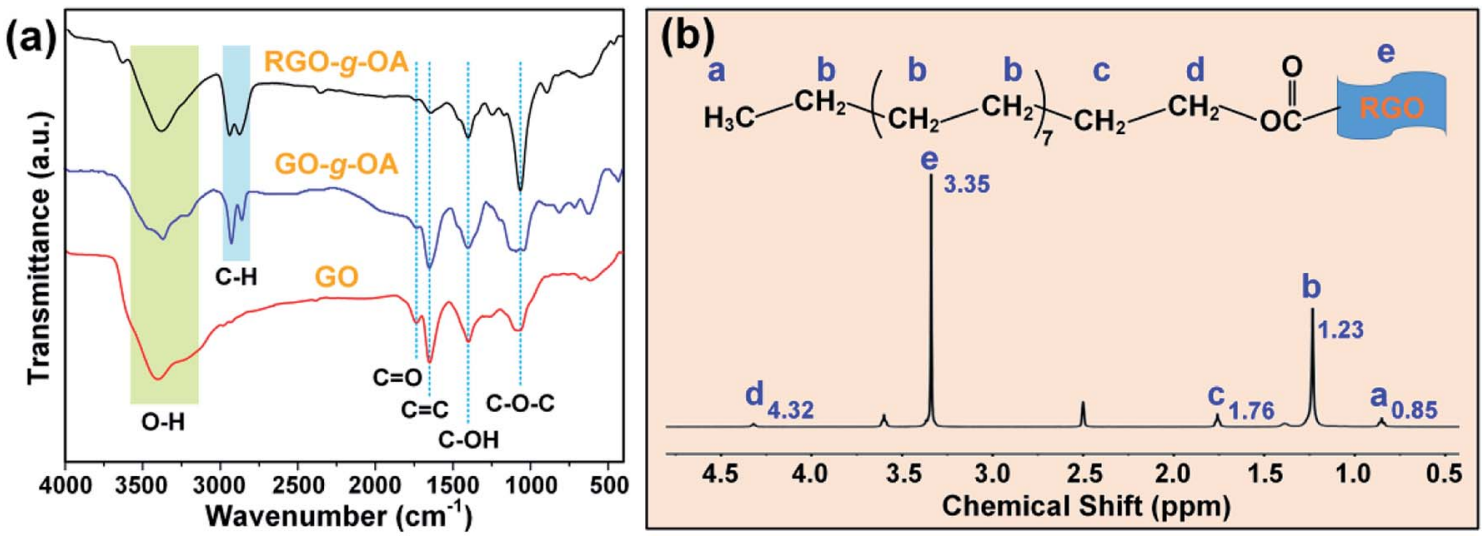

Fig. 1 (a) FTIR spectra of GO, GO-g-OA, RGO-g-OA; (b) ${ }^{1} \mathrm{H}$ NMR spectrum of RGO-g-OA in deuterated DMSO (chemical shift, 2.5 ppm). 
the disordered restacking of mildly reduced GO sheets during the grafting process. For RGO-g-OA, the (002) diffraction peak shifts to $2 \theta=24.3^{\circ}$, corresponding to $d$-spacing of $0.362 \mathrm{~nm}$, which is less than that of GO- $g$-OA, implying that the GO- $g$-OA was reduced after the chemical reduction by L-ascorbic acid. Note that the (001) peak would disappear because of delamination if GO was fully transformed into RGO. Thus, in this work, the strong (001) peak remaining signifies partial/incomplete reduction of GO. Overall, it is reasonable to infer that specific hydrophobic/highly-oleophilic structure of RGO-g-OA featuring large hydrophobic graphite-like domains in the middle region and long-chain alkane groups anchored onto the edge of GO sheets was acquired after the grafting reaction and chemical reduction procedures. ${ }^{36,37}$

\subsection{Microstructural morphology and particle size characterization}

Microstructural morphology and particle size of GO-based lubricating additives usually play vital roles in their dispersion capacities in the lubricant matrixes and in lubrication performance. ${ }^{4,17,31}$ Thus, TEM and DLS measurements of GO and RGO- $g$-OA were performed in this work. From the TEM images of GO (Fig. 3a), it can be clearly observed that GO nanoflakes are highly transparent with some folds and ripples at the edges, implying a very small thickness of GO as a result of the highlyefficient oxidation exfoliation course. Compared with GO, RGO$g$-OA flakes display a more crumpled and stacked morphology and the agglomeration is more severe than GO, which may be
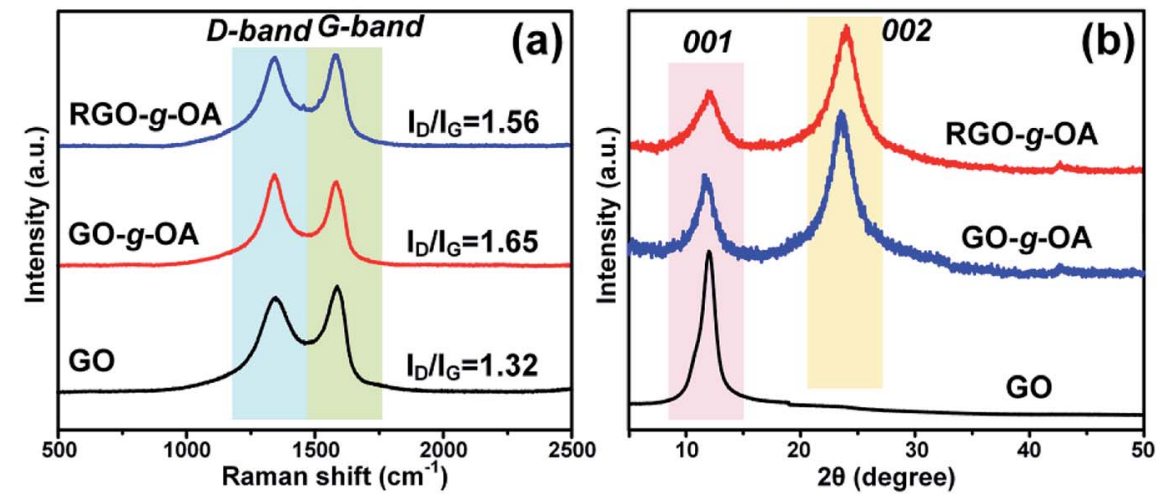

Fig. 2 Raman spectra (a) and XRD patterns (b) of GO, GO-g-OA, and RGO-g-OA.
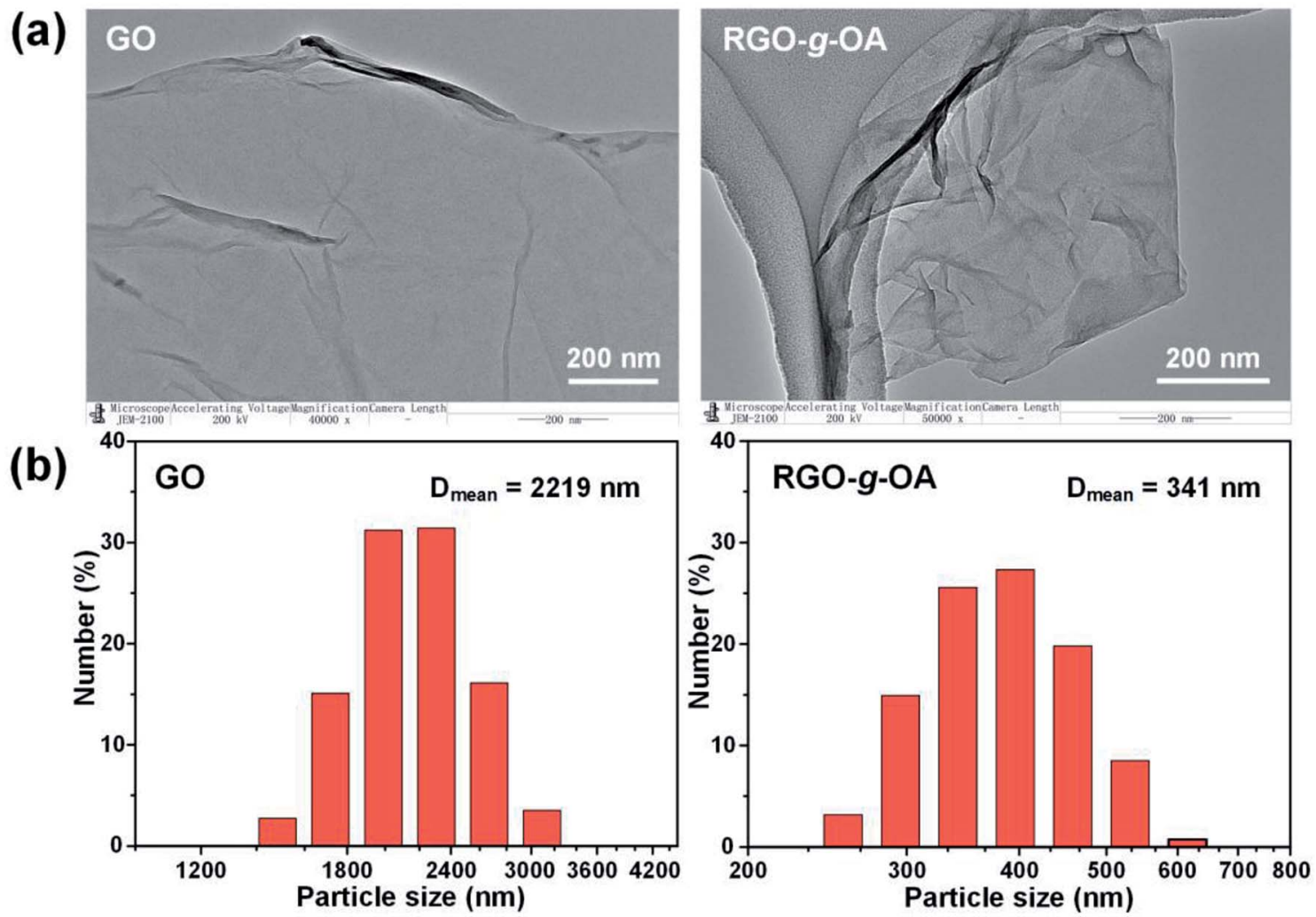

Fig. 3 TEM images (a) and particle size distributions (b) of GO and RGO-g-OA. 
attributed to the increasing van der Waals force interactions between graphene layers due to effective removal of most oxygenated groups being associated with decreased interlayer distances, as evidenced in XRD results. ${ }^{38}$

Additionally, it is found that particle size distribution of GO mainly ranges from 1400 to $3200 \mathrm{~nm}$, while the particle sizes of RGO-g-OA vary from 250 to $650 \mathrm{~nm}$; the average particle sizes of GO and RGO-g-OA are 2219 and $341 \mathrm{~nm}$, respectively (Fig. 3b). Such a smaller particle size for RGO- $g$-OA can be attributed to effective mechanical crushing from the high-speed dispersion and strong shear forces.

\subsection{Thermal and dispersion stability}

To evaluate the thermal stability of GO-based lubricating oil additives, TGA measurements of GO, GO- $g$-OA, and RGO- $g$-OA were performed. As indicated in Fig. 4a, for neat GO, about 3\% weight loss can be observed for the first stage (I) of decomposition below $100{ }^{\circ} \mathrm{C}$, due to the adsorbed water. Next, $25 \%$ weight loss is found in the second stage (II) ranging from 100 to $265{ }^{\circ} \mathrm{C}$, mainly attributed to the removal of large amounts of oxygenated functional groups in GO. The weight loss of GO in the third stage (III) from 265 to $460{ }^{\circ} \mathrm{C}$ is determined to be around $5 \%$, which may be ascribed to the removal of other, more stable functional groups.

Compared with GO, both GO- $g$-OA and RGO-g-OA show almost negligible weight decrease in stage I, indicating that the moisture content is very low in these two materials. The weight losses of GO-g-OA and RGO- $g$-OA in stage II are $14 \%$ and $13 \%$, respectively, which is due to the decomposition of oxygencontaining functional groups. It is noteworthy that the weight loss of RGO- $g$-OA at this temperature range is slightly lower than that of GO- $g$-OA, implying that the amount of oxygen-containing functional groups on RGO-g-OA decreases after the chemical reduction process. Moreover, the weight losses of GO- $g$-OA and RGO-g-OA in stage III are about $16 \%$ and $17 \%$, respectively, which can be ascribed to the decomposition of long-chain alkanes. The total weight losses of GO-g-OA and RGO-g-OA in the measurement range are $37 \%$ and $35 \%$, respectively, which are less than that of GO $(41 \%)$. These results clearly demonstrate that successful grafting of OA accompanied by subsequent chemical reduction endow RGO- $g$-OA with much higher thermal stability.

It is well established that durable dispersion stability of additives in lubricants is a prerequisite for their efficient lubrication performance. The dispersion stability of GO-based additives in the finished oil was examined using two testing methods (standing and intensified centrifugation) and results are presented in Fig. 5. In the current study, high-performance homemade finished oil was utilized as the lubricant base oil. As indicated in Fig. 5a, the additives GO, GO- $g$-OA and RGO- $g$-OA can be uniformly dispersed in the finished oil (mass fraction, $0.01 \mathrm{wt} \%$ ) with the aid of sonication. Obviously, GO additives containing abundant oxygenated groups associated with ultralow lipophilicity display severe agglomeration and become fully sedimentary after standing for one month, revealing inferior dispersion stability in the finished oil. In comparison, GO- $g$-OA exhibited a greatly improved dispersion capacity with a slight stratification phenomenon, which suggested the high efficacy of long-chain alkane functionalization toward the dispersibility of GO-based additives. More importantly, RGO- $g$-OA nanomaterials retained a superior dispersion stability even after one month. Strengthened centrifugal force assessment also displays similar results (Fig. 5b), that is, both RGO and GO- $g$-OA additives tend to agglomerate and sediment from the lubricant substrate, whereas the RGO-g-OA possesses excellent dispersion stability with no obvious precipitation behavior. Fig. $\mathrm{S} 1 \uparrow$ further demonstrates that RGO- $g$-OA possesses good dispersion stability in an elevated temperature environment (such as $100{ }^{\circ} \mathrm{C}$ ), implying great prospects for practical application. Such remarkable dispersion durability of RGO- $g$-OA is believed to originate from the synergistic effect between the significantly improved lipophilicity of graphene additives, arising from long-chain alkyl (OA) grafting and partial chemical reduction, and the small-size effect (RGO- $g$ OA, $\left.D_{\text {mean }}=341 \mathrm{~nm}\right)$.

\subsection{Tribological performance}

Inspired by the excellent dispersibility of RGO- $g$-OA in the finished oil, its tribological properties were further investigated.

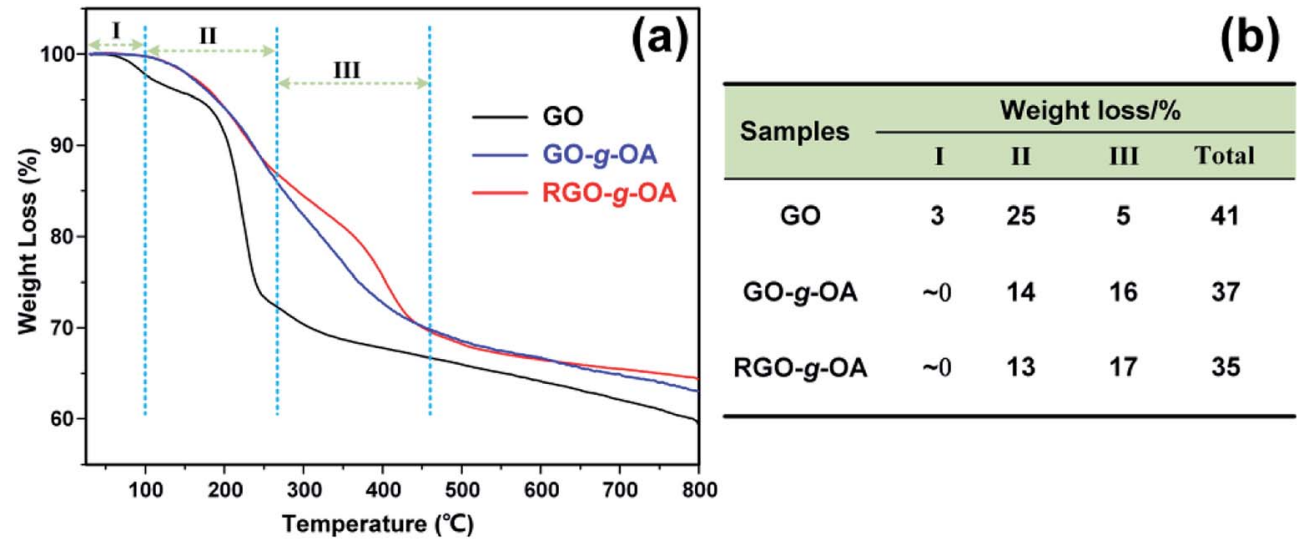

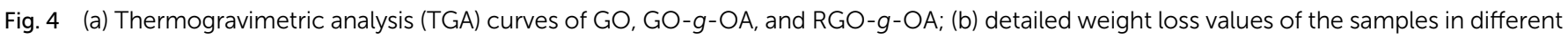
decomposition temperature ranges. 


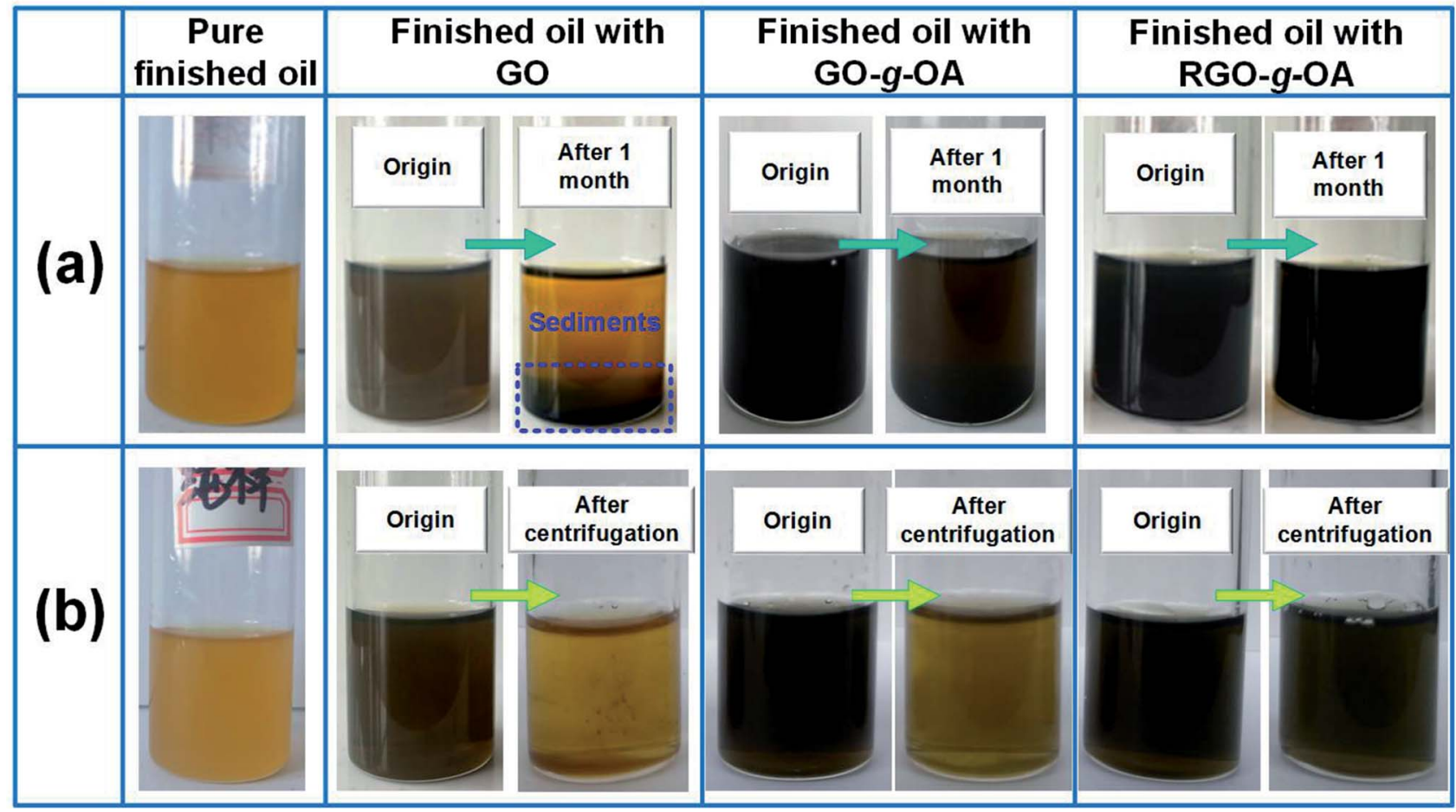

Fig. 5 Dispersion stability of the finished oils with different GO-based additives. Digital images of finished oil dispersed with GO, GO- $g$-OA, and RGO-g-OA before and after standing for one month (a); before and after intensified centrifugation operation (supernatants) under 8000 rpm for $15 \mathrm{~min}$ (b). The mass fraction of each additive in lubricants is $0.01 \mathrm{wt} \%$.

Fig. 6a shows the typical friction coefficient variations of finished oil containing different mass fractions of RGO-g-OA as a function of time. Apparently, pure finished oil exhibits a friction coefficient of $\sim 0.072$. Interestingly, the finished oil with RGO-g-OA nanoadditives displays a lower friction coefficient than that of the pristine finished oil. This result clearly indicates that RGO-g-OA nanolubricant can effectively enhance the tribological properties of finished oil. Note that the enhanced tribological performance of RGO-g-OA in pristine finished oil is strongly influenced by its weight fraction. As shown in Fig. 6b, with $0.005 \mathrm{wt} \%$ RGO-g-OA in the base oil, the friction coefficient of the composite finished oil is greatly reduced and obtains the lowest value of 0.065 . In other words, the finished oil filled with 0.005 wt $\%$ RGO-g-OA achieves the highest friction-reduction performance. However, when further increasing the mass fractions of RGO-g-OA, the friction coefficient also increases. For example, when the mass fraction of RGO-g-OA nanomaterial additive reaches $0.025 \mathrm{wt} \%$, the friction coefficient acquires a value of 0.076 , which is higher than that of the pristine finished oil (0.072). This phenomenon may arise from the fact that excess RGO-g-OA additives pile up between friction pairs and block the oil film, leading to abrasive-like wear (dry friction) and thus inferior lubrication performance. ${ }^{20,28}$ Notably, with increase of the normal load from 100 to $150 \mathrm{~N}$, the finished oil with $0.005 \mathrm{wt} \%$ RGO-g-OA shows a distinct improvement in friction-reducing property compared to that of finished oil (Fig. S2, ESI $\dagger$ ). To exclude the effect of nanoparticle additives in finished oil, pure base oil (hydraulic oil) with almost no additives is used as the control lube oil in tribological performances.
As shown in Fig. 6(c and d), the base oil shows a friction coefficient as high as 0.30 , which is greatly higher than that of finished oil. Encouragingly, the base oil with RGO-g-OA additives dispersed at an ultralow concentration (0.005 wt\%) achieves a significantly reduced friction coefficient of 0.24 compared with the pure base oil, indicating the superior friction-reduction performance of RGO-g-OA additives.

In addition to the remarkable friction reduction effect, excellent wear-resistance performance of RGO-g-OA nanolubricant was also observed in our current work. Fig. 7 and Table 1 present the surface morphologies, cross-sectional height profiles, and volumes of the wear tracks on the surfaces lubricated with pristine finished oil and various RGO- $g$ OA filled finished oils. Obviously, for the worn surface of pure finished oil, as shown in Fig. 7a, a distinct dumbbell-like wear track can be observed. Meanwhile, the cross-sectional height profiles of the wear track (Fig. 7b) display dramatic fluctuation, which may be due to the extrusion and deformation effects between the measured ball and steel disc. A wear volume of $18354 \mu \mathrm{m}^{3}$ is achieved for the steel disc lubricated with pure finished oil. In contrast, the steel disks lubricated by the finished oil filled with RGO-g-OA nanoadditives display decreased wear track areas, wear heights (depth), and wear volumes compared with those of pure finished oil. These characteristic parameters exhibit similar variation trends as the friction coefficients related to the RGO- $g$-OA mass fractions in the finished oil. Interestingly, the finished oil containing $0.005 \mathrm{wt} \%$ RGO-g-OA achieves the best anti-wear performances: (a) a quite weak and blurry wear area; (b) a slight fluctuation 

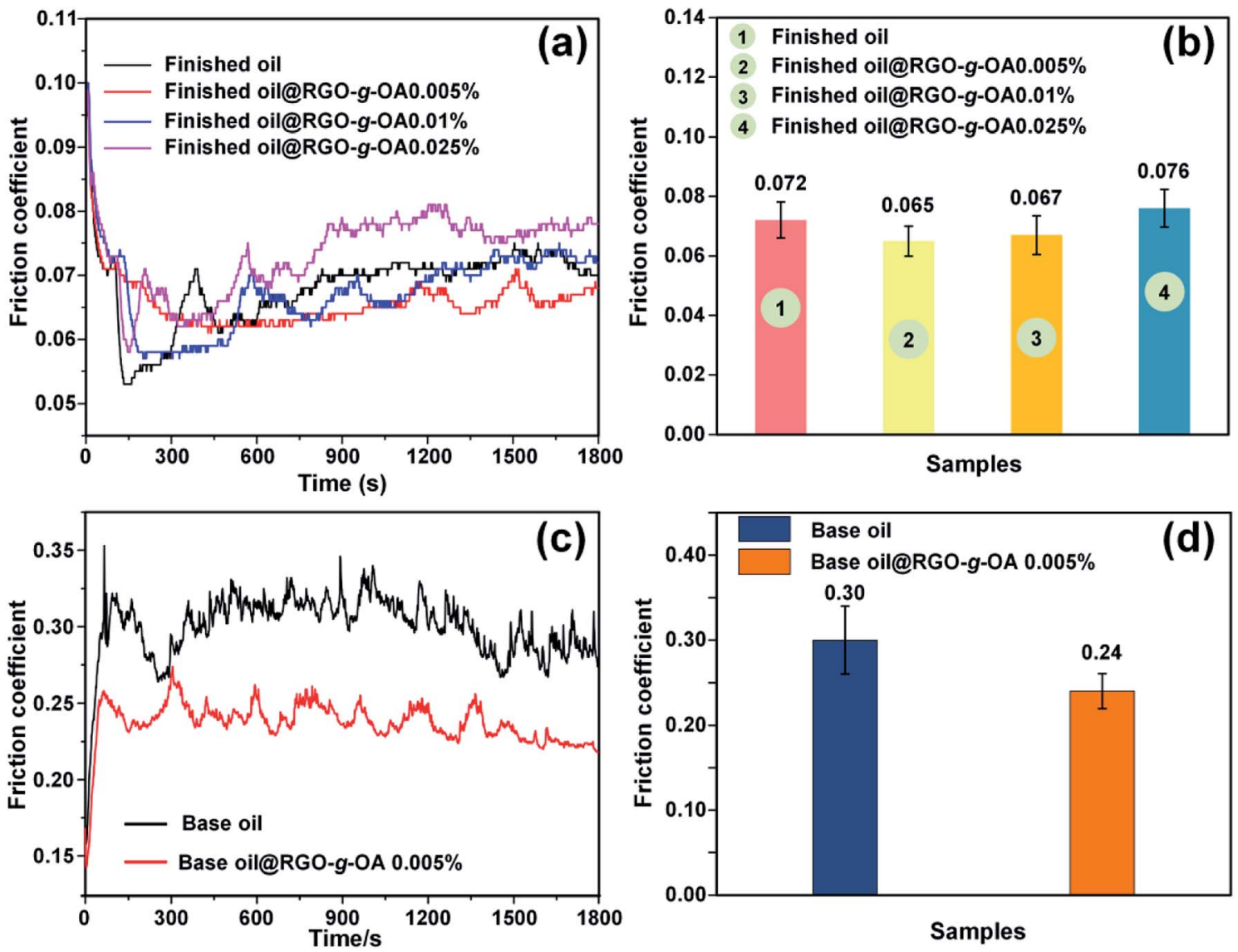

Fig. 6 Typical friction coefficients over time ( $a$ and $c$ ) and average friction coefficients ( $b$ and d) of pristine finished oil, base oil and their counterparts filled with different mass contents of RGO-g-OA on steel discs; the applied load is $100 \mathrm{~N}$.

(and lower wear depth) on the cross-sectional height profile of the wear track; (c) the smallest wear volume value of $10316 \mu \mathrm{m}^{3}$, which was decreased by $44 \%$ compared to the pristine finished oil. When the load was increased from 100 to $150 \mathrm{~N}$, the finished oil with $0.005 \mathrm{wt} \%$ RGO-g-OA still showed good anti-wear performance compared to finished oil (Fig. S3 and Table S2,
ESI $\dagger$ ). The incorporation of $0.005 \mathrm{wt} \%$ RGO-g-OA into pure base oil (Fig. S4 and Table S3, ESI†) further demonstrates its high effectiveness in wear-resistance performance. Note that when the RGO- $g$-OA additive content reaches $0.025 \mathrm{wt} \%$, the wear volume presents a significant increase and achieves a value as high as $31639 \mu \mathrm{m}^{3}$, increased by $72 \%$ compared to the pristine (a)
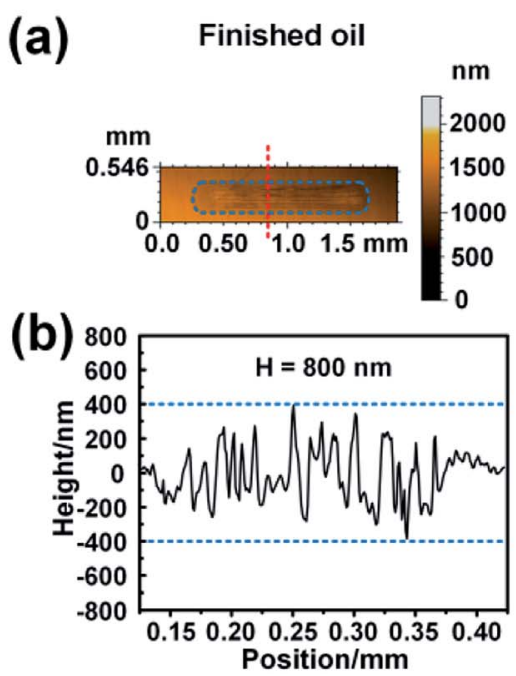

Finished oil@RGO$g-0 A 0.005 \%$
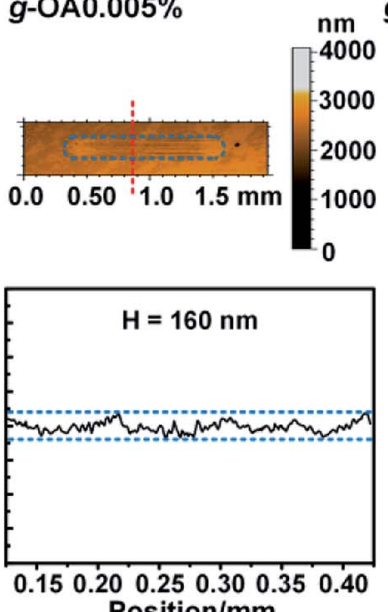

Finished oil @ RGOg-OA0.01\%
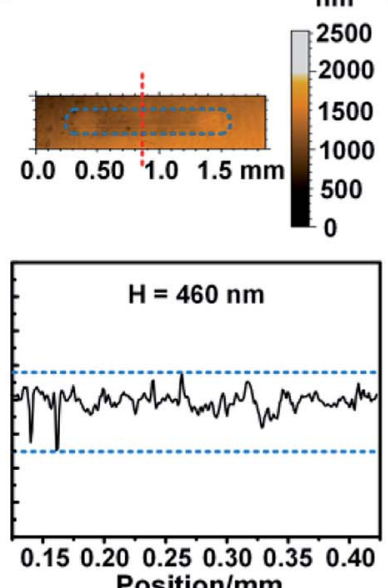

Finished oil@RGO-
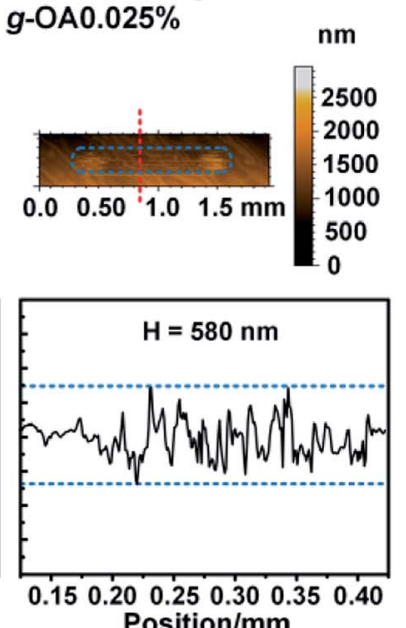

Fig. 7 (a) Surface morphologies of the worn regions on the steel disks after friction tests lubricated with the finished oil and finished oil filled with different contents of RGO-g-OA; (b) the average cross-sectional height profiles of the dashed line areas labeled in (a). 
Table 1 Wear volumes of the worn surfaces on the steel discs under load of $100 \mathrm{~N}$

\begin{tabular}{llc}
\hline Samples & Wear volume $\left(\mu \mathrm{m}^{3}\right)$ & Increment \\
\hline Finished oil & 18354 & $0 \%$ \\
Finished oil@RGO-g-OA 0.005\% & 10316 & $-44 \%$ \\
Finished oil@RGO-g-OA 0.010\% & 11664 & $-36 \%$ \\
Finished oil@RGO-g-OA 0.025\% & 31639 & $+72 \%$
\end{tabular}

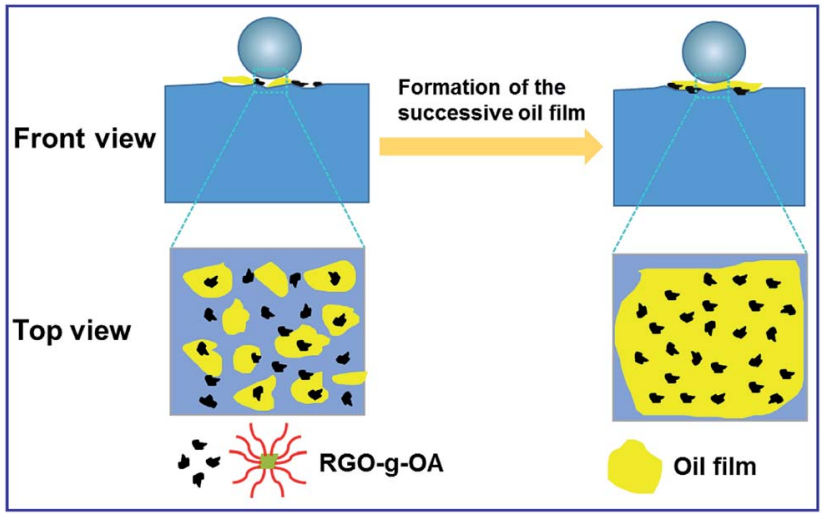

Fig. 8 Schematic of friction reduction mechanism of the finished oil with added $\mathrm{RGO}-\mathrm{g}-\mathrm{OA}$.

finished oil. Such a poor wear-resistance property is believed to originate from the excess RGO-g-OA additives, as discussed above. Consequently, in combination with the friction-reducing property, it is indisputable that the introduction of RGO-g-OA in finished oil at an extremely low amount $(0.005 \mathrm{wt} \%)$ greatly improves both the friction reduction and anti-wear performances, demonstrating its intriguing prospect as a highefficiency lubricant nanoadditive.

A mechanism for the excellent friction-reducing and antiwear properties of RGO- $g$-OA nanomaterial was tentatively proposed and is illustrated in Fig. 8. Due to the small-size and ultrathin structural characteristics of RGO-g-OA nanosheets, as depicted in Fig. 3, they easily enter the rubbing interfaces between the ball and steel disc, thereby promoting the lubricating oil to form a discontinuous, thin and unstable oil film at the initial stage of the friction process. However, after a short run-in period, RGO-g-OA nanoadditives fill the pits and grooves formed on the steel disc, facilitating formation of a continuous, thick and stable tribological film to avoid direct contact between the rubbing steel ball and disc (as evidenced by SEM and Raman spectra results in Fig. S5†). Benefiting from the filling and stable tribological shielding film, the finished oil with dispersed RGO-g-OA nanolubricants endows surfaces with superior friction-reducing and wear resistance performances.

\section{Conclusions}

In brief, we successfully fabricated a small-size, long-chain alkylated reduced graphene oxide (RGO- $g$-OA) nanoparticle additive with high dispersibility and remarkable lubrication properties by a facile integration methodology composed of mechanical crushing, surface grafting, and subsequent chemical reduction. Thanks to the effective introduction of oleophilic long-chain alkyls onto GO and the miniaturized size effect, the resulting RGO-g-OA nanocomposite achieved excellent dispersion capability and stability in the finished oil. The thermal stability of RGO- $g$-OA was also improved to some extent due to the covalent surface functionalization of GO. Furthermore, the finished oil formulated with RGO-g-OA nanoadditives at an ultralow content of $0.005 \mathrm{wt} \%$ achieved remarkable friction reduction and anti-wear performances. Specifically, the corresponding friction coefficient and wear volume were dramatically decreased to 0.065 and $10316 \mu \mathrm{m}^{3}$, by $9.7 \%$ and $44 \%$, respectively, in comparison with those of pristine finished oil. Such outstanding lubrication properties should be attributed to the fact that the ultrafine RGO-g-OA can form an intact protective tribology film between rubbing surfaces to prevent them from direct contact, thereby reducing friction and wear during the friction process. These observations demonstrate the highly-desirable effectiveness of RGO-g-OA nanocomposite for enhanced dispersibility and suggest its utilization as an efficient lubricant additive toward high-performance tribological applications.

\section{Conflicts of interest}

There are no conflicts to declare.

\section{Acknowledgements}

The authors are particularly grateful for the financial support from the National Natural Science Foundation of China (Grant No. 51703113 and 51703111), the Shandong Provincial Natural Science Foundation (Grant No. ZR2017BEM039, ZR2017BEM011, and ZR2016XJ001), and China Postdoctoral Science Foundation (2018M630763).

\section{References}

1 S. Bagheri, N. Jamal, A. Halilu and A. TermehYousefi, Sci. Rep., 2018, 8, 6221.

2 K. Holmberg and A. Erdemir, Friction, 2017, 5, 263-284.

3 V. W. Wong and S. C. Tung, Friction, 2016, 4, 1-28.

4 X. Dou, A. R. Koltonow, X. He, H. D. Jang, Q. Wang, Y. Chung and J. Huang, Proc. Natl. Acad. Sci. U. S. A., 2016, 113, 15281533.

5 Z. Wang, R. Ren, H. Song and X. Jia, Appl. Surf. Sci., 2018, 428, 630-639.

6 P. Rabaso, F. Dassenoy, F. Ville, M. Diaby, B. Vacher, T. Le Mogne, M. Belin and J. Cavoret, Tribol. Lett., 2014, 55, 503516.

7 D. Berman, A. Erdemir and A. V. Sumant, Mater. Today, 2014, 17, 31-42.

8 A. K. Rasheed, M. Khalid, W. Rashmi, T. C. S. M. Gupta and A. Chan, Renewable Sustainable Energy Rev., 2016, 63, 346362. 
9 D. Berman, A. Erdemir and A. V. Sumant, Carbon, 2013, 54, 454-459.

10 D. Berman, A. Erdemir and A. V. Sumant, Carbon, 2013, 59, 167-175.

11 V. Eswaraiah, V. Sankaranarayanan and S. Ramaprabhu, ACS Appl. Mater. Interfaces, 2011, 3, 4221-4227.

12 J. Zhao, Y. Li, Y. Wang, J. Mao, Y. He and J. Luo, RSC Adv., 2017, 7, 1766-1770.

13 J. Zhao, Y. He, Y. Wang, W. Wang, L. Yan and J. Luo, Tribol. Int., 2016, 97, 14-20.

14 J. Lin, L. Wang and G. Chen, Tribol. Lett., 2011, 41, 209-215.

15 V. Georgakilas, M. Otyepka, A. B. Bourlinos, V. Chandra, N. Kim, K. C. Kemp, P. Hobza, R. Zboril and K. S. Kim, Chem. Rev., 2012, 112, 6156-6214.

16 S. Li, Q. Li, R. W. Carpick, P. Gumbsch, X. Z. Liu, X. Ding, J. Sun and J. Li, Nature, 2016, 539, 541-545.

17 J. Mao, J. Zhao, W. Wang, Y. He and J. Luo, Tribol. Int., 2018, 119, 614-621.

18 H. Xiao and S. Liu, Mater. Des., 2017, 135, 319-332.

19 J. B. Pu, L. P. Wang and Q. J. Xue, Tribology, 2014, 34, 93-112.

20 H. Song, Z. Wang, J. Yang, X. Jia and Z. Zhang, Chem. Eng. J., 2017, 324, 51-62.

21 A. Chouhan, H. P. Mungse, O. P. Sharma, R. K. Singh and O. P. Khatri, J. Colloid Interface Sci., 2018, 513, 666-676.

22 A. Wolk, M. Rosenthal, S. Neuhaus, K. Huber, K. Brassat, J. K. N. Lindner, R. Grothe, G. Grundmeier, W. Bremser and R. Wilhelm, Sci. Rep., 2018, 8, 5843.

23 L. Zhang, Y. He, L. Zhu, C. Yang, Q. Niu and C. An, Ind. Eng. Chem. Res., 2017, 56, 9029-9034.

24 Z. Cheng, W. Li, P. Wu and Z. Liu, Ind. Eng. Chem. Res., 2017, 56, 5527-5534.
25 J. Hong, E. Sohn, S. Park and H. S. Park, Chem. Eng. J., 2015, 269, 229-235.

26 Y. Liu, Y. L. Zhang, Y. Liu, H. Jiang, D. Han, B. Han, J. Feng and H. B. Sun, Chem. Rec., 2015, 16, 1244-1255.

27 H. Hu, C. C. K. Allan, J. Li, Y. Kong, X. Wang, J. H. Xin and H. Hu, Nano Res., 2014, 7, 418-433.

28 N. A. Ismail and S. Bagheri, Mater. Sci. Eng., B, 2017, 222, 3442.

29 S. Kumari, O. P. Sharma, R. Gusain, H. P. Mungse, A. Kukrety, N. Kumar, H. Sugimura and O. P. Khatri, ACS Appl. Mater. Interfaces, 2015, 7, 3708-3716.

30 S. Choudhary, H. P. Mungse and O. P. Khatri, J. Mater. Chem., 2012, 22, 21032-21039.

31 Z. L. Cheng, W. Li, P. R. Wu and Z. Liu, J. Alloys Compd., 2017, 722, 778-784.

32 Z. Liu, K. Nørgaard, M. H. Overgaard, M. Ceccato, D. M. A. Mackenzie, N. Stenger, S. L. S. Stipp and T. Hassenkam, Carbon, 2018, 127, 141-148.

33 B. S. Lee, Y. Lee, Y. H. Jun and Y. C. Choi, Carbon Lett., 2015, 16, 255-259.

34 S. Zhao, S. Xie, Z. Zhao, J. Zhang, L. Li and Z. Xin, ACS Sustainable Chem. Eng., 2018, 6, 7652-7661.

35 M. K. Balyan, Acta Crystallogr., Sect. A: Found. Adv., 2018, 74, 204-215.

36 R. Xiong, H. S. Kim, L. Zhang, V. F. Korolovych, S. Zhang, Y. G. Yingling and V. V. Tsukruk, Angew. Chem., Int. Ed., 2018, 57, 8508-8513.

37 X. Fan, W. Peng, Y. Li, X. Li, S. Wang, G. Zhang and F. Zhang, Adv. Mater., 2008, 20, 4490-4493.

38 N. Sarlak and T. J. Meyer, J. Mol. Liq., 2017, 243, 654-663. 